\title{
A Characterization of the Extended Serial Correspondence*
}

\author{
Eun Jeong Heo ${ }^{\dagger}$ and Özgür Yılmaz
}

December 10, 2013

\begin{abstract}
We study the problem of assigning objects to a group of agents. We focus on probabilistic methods that take agents' ordinal preferences over the objects. Importantly, we allow for indifferences among objects. Katta and Sethuraman (2006) propose the extended serial correspondence to solve this problem. Our main result is a characterization of the extended serial correspondence in welfare terms by means of stochastic dominance efficiency, stochastic dominance no-envy and "limited invariance," a requirement we adapt from Heo (2011). We also prove that an assignment matrix is selected by the extended serial correspondence if and only if it satisfies "non-wastefulness" and "ordinal fairness," which we adapt from Kesten et al. (2011).

JEL classification: C70, D61, D63.
\end{abstract}

Keywords: the serial rule; sd-efficiency; sd no-envy; the extended serial correspondence; limited invariance.

${ }^{*}$ This work is obtained by merging independent papers by the two authors. The current manuscript is developed from Heo and Yllmaz (2011).

${ }^{\dagger}$ Department of Economics, University of Rochester, Rochester NY 14627, USA and Department of Economics, University of Bonn, Bonn 53113, Germany (heoeunjeong@gmail.com)

${ }^{\ddagger}$ College of Administrative Sciences and Economics, Koç University, Sarıyer, Istanbul, 34450, Turkey (ozyilmaz@ku.edu.tr)

We are indebted to William Thomson for his guidance and support. Heo is grateful to Anna Bogomolnaia for useful comments. We benefited from comments from the seminar participants at Columbia University, in particular, Yeon-koo Che, Fuhito Kojima, and Jay Sethuraman, the participants at the Frontiers of Market Design Conference at Monte Verità, in particular, Eric Budish and M. Utku Ünver. All errors are our own responsibility. 


\section{Introduction}

We study the problem of assigning indivisible goods, or "objects," to a group of agents. We focus on probabilistic solutions that take agents' ordinal preferences over the objects as input. Importantly, we allow for indifferences among objects, that is, agents may have "weak preferences" over objects. Agents receive random assignments which are the probability distributions over the objects.

Given an ordinal preference over objects, a partial order over random assignments can be derived on the basis of first-order stochastic dominance (throughout the paper, the notation "sd" stands for "stochastic dominance" ${ }^{1}$ ). Two central requirements are proposed by using this relation (Bogomolnaia and Moulin, 2001). First, "sd efficiency" requires that there should be no Pareto improvement in sd sense. Second, "sd no-envy" requires that each agent should find his own assignment at least as desirable as that of each other agent in sd sense (the formal definitions are available in Section 2).

The "serial rule" (Bogomolnaia and Moulin, 2001) has been extensively studied in the literature, as a rule satisfying these two requirements. ${ }^{2}$ It is also the only one to satisfy the two requirements together with one of several invariance requirements. These characterizations are first provided by Hashimoto and Hirata (2011), Heo (2011), and Kesten et al. (2011). Heo (2011) introduces "limited invariance" and a weaker fairness requirement in place of sd no-envy. Hashimoto et al. (2013) adopts limited invariance, sharpening the result in Bogomolnaia and Heo (2012). They also provide a result that does not involve any invariance-type axiom. ${ }^{3}$

In presence of weak preferences, however, the implication of sd efficiency and sd no-envy is not obvious due to the difficulties generated by indifference. This problem is solved by Katta and Sethuraman (2006). For each (weak) preference profile, they introduce a sequence of flow graphs and then calculate the maximal flow of each graph. The resulting sequence of maximal flows determines a set of assignment matrices. The "extended serial correspondence" is defined through this algorithm. They show that it is "essentially single-valued" and satisfies sd efficiency and sd no-envy.

In this paper, we provide two characterizations of the extended serial correspondence. The first result is by means of sd-efficiency, sd no-envy, and "limited invariance," a natural adaptation to the domain of weak preferences of the requirement of the same name in Heo (2011). This result is an extension of Hashimoto et al. (2013). ${ }^{4}$

We present another characterization by means of "non-wastefulness" and "ordinal fairness,"

\footnotetext{
${ }^{1}$ We adopt the terminology and notation of Thomson (2010a, 2010b).

${ }^{2}$ The authors call it "probabilistic serial mechanism".

${ }^{3}$ This paper is developed from merging Hashimoto and Hirata (2011) and Kesten et al. (2011).

${ }^{4}$ Note that, when applied to the strict preference profiles, these requirements coincide with those in Hashimoto et al. (2013) and the extended serial correspondence narrows down to the serial rule. We discuss why this result is logically stronger in Section 3.
} 
both requirements being adapted from Kesten et al. (2011). An alternative representation of assignments, called "preference-decreasing consumption schedules," allows us to obtain a straightforward proof. Using this representation, we invoke an important structural property of assignment matrices selected by the extended serial correspondence (Lemma 7). ${ }^{5}$

This paper is organized as follows. Section 2 introduces model and requirements. Section 3 presents our main result. Section 4 presents our second characterization result.

\section{Model}

Let $A \equiv\left\{a_{1}, \cdots, a_{m}\right\}$ be a set of objects and $N \equiv\{1,2, \cdots, n\}$ a set of agents. We assume that $|N| \leq|A|{ }^{6}{ }^{6}$ There may be multiple copies of each object. For each $i \in N, R_{i}$ represents agent $i$ 's weak preferences over $A$. For each $i \in N$ and each pair $a, b \in A$, we write that $a I_{i} b$ if and only if $a R_{i} b$ and $b R_{i} a$. Derive a strict preference over $A$ for $R_{i}$ - let us call it $P_{i}$ - as follows: for each pair $a, b \in A, a P_{i} b$ if and only if $a R_{i} b$ and $\neg\left(a I_{i} b\right)$. For each pair of disjoint $B, B^{\prime} \subseteq A$, we write $B P_{i} B^{\prime}$ if and only if for each $b \in B$ and each $b^{\prime} \in B^{\prime}, b P_{i} b^{\prime}$ ( $B R_{i} B^{\prime}$ and $B I_{i} B^{\prime}$ are defined similarly). Let $\mathcal{R}$ be the set of weak preferences over $A$ and $\mathcal{P}$ be the set of strict preferences over $A$. For each $i \in N$, each $R_{i} \in \mathcal{R}$, and each $B \subseteq A$, let $\left.R_{i}\right|_{B}$ be the preference $R_{i}$ restricted to $B$ and $C h\left(R_{i}, B\right)$ be the set of objects in $B$ that agent $i$ most prefers. For each $M \subseteq N$, let $C h\left(R_{M}, B\right) \equiv \bigcup_{i \in M} C h\left(R_{i}, B\right)$. For each $R \in \mathcal{R}^{N}$, each $i \in N$, and each $a \in A$, let $U\left(R_{i}, a\right) \equiv\left\{b \in A: b R_{i} a\right\}$ be the weak upper contour set at $a$ of $R_{i}$. Also, let $I\left(R_{i}, a\right) \equiv\left\{b \in A: a I_{i} b\right\}$ be the indifference class of $a$ at $R_{i}$. For each $B \subseteq A$, $U\left(R_{i}, B\right) \equiv \bigcup_{a \in B} U\left(R_{i}, a\right)$.

An economy is a list $(A, N, R)$. We fix $A$ and $N$, so the notation for an economy can be simplified to a preference profile $R$. Let $\mathcal{R}^{N}$ be the set of all economies. An assignment matrix is an $|N| \times|A|$ matrix $\pi \equiv\left(\pi_{i a}\right)_{i \in N, a \in A}$, where $\pi_{i a}$ is the probability of agent $i$ receiving $a$, such that (i) for each $i \in N$ and each $a \in A, \pi_{i a} \in[0,1]$, (ii) for each $a \in A, \sum_{i \in N} \pi_{i a} \leq 1$, and (iii) for each $i \in N, \sum_{a \in A} \pi_{i a}=1$. An assignment matrix $\pi \in \Pi$ is deterministic if for each $i \in N$ and each $a \in A, \pi_{i a} \in\{0,1\}$. By the Birkhoff-von Neumann theorem (Birkhoff (1946), von Neumann (1953)), each assignment matrix can be written as a convex combination of deterministic assignment matrices. Let $\Pi$ be the set of all assignment matrices. For each $B \subseteq A$ and each pair $\pi, \pi^{\prime} \in \Pi$, we say that $\boldsymbol{\pi}$ and $\boldsymbol{\pi}^{\prime}$ coincide on $\boldsymbol{B}$ if for each $i \in N$ and each $a \in B, \pi_{i a}=\pi_{i a}^{\prime}$. Let $2^{\Pi}$ be the collection of all subsets of $\Pi$. Let $\phi$ be the empty set. A rule is a mapping $\varphi: \mathcal{R} \rightarrow \Pi$. A correspondence is a mapping $\Phi: \mathcal{R} \rightarrow 2^{\Pi} \backslash\{\phi\}$. A subcorrespondence $\Psi$ of $\Phi$ is a correspondence such that for each $R \in \mathcal{R}^{N}, \Psi(R) \subseteq \Phi(R)$.

Let $R \in \mathcal{R}^{N}$. Let $\pi, \pi^{\prime} \in \Pi$. For each $i \in N$, we say that $\boldsymbol{\pi}_{\boldsymbol{i}}$ weakly stochastically

\footnotetext{
${ }^{5}$ Note that these two requirements are "punctual", that is, they apply to each economy separately. Heo (2013) also characterizes the same solution by means of two punctual requirements on a restricted domain of preference profiles.

${ }^{6}$ Otherwise, we may introduce a "null object" (with $n$ copies) which represents "receiving no object".
} 
dominates $\boldsymbol{\pi}_{\boldsymbol{i}}^{\prime}$ at $\boldsymbol{R}_{\boldsymbol{i}}$, if for each $a \in A, \sum_{b \in U\left(R_{i}, a\right)} \pi_{i b} \geq \sum_{b \in U\left(R_{i}, a\right)} \pi_{i b}^{\prime}$. If there is at least one strict inequality, we say that $\pi_{i}$ stochastically dominates $\pi_{i}^{\prime}$ at $\boldsymbol{R}_{i}$. We say that $\boldsymbol{\pi}$ stochastically dominates $\boldsymbol{\pi}^{\prime}$ at $\boldsymbol{R}$, if for each $i \in N, \pi_{i}$ weakly stochastically dominates $\pi_{i}^{\prime}$ at $R_{i}$ and for some $i \in N, \pi_{i}$ stochastically dominates $\pi_{i}^{\prime}$ at $R_{i}$. Throughout the paper, the notation "sd" stands for "stochastic dominance."

The following are requirements of assignment matrices based on the sd relations. Consider an assignment matrix and a preference profile. First is an efficiency requirement. There should be no other assignment matrix at which some agent is sd better off and no agent is sd worse off. Formally, $\pi$ is sd-efficient at $\boldsymbol{R}$ if there is no $\pi^{\prime} \in \Pi$ such that for each $i \in N$, $\pi_{i}^{\prime}$ weakly stochastically dominates $\pi_{i}$ at $R_{i}$ and for some $i \in N, \pi_{i}^{\prime}$ stochastically dominates $\pi_{i}$ at $R_{i}$. The corresponding requirement of a correspondence $\Phi$ is as follows:

Sd efficiency: For each $R \in \mathcal{R}^{N}$ and each $\pi \in \Phi(R), \pi$ is sd-efficient at $R$.

The following is a fairness requirement: each agent should find his assignment at least as "sd-desirable" as that of each other agent. Formally, $\pi$ is sd envy-free at $\boldsymbol{R}$ if for each pair $i, j \in N, \pi_{i}$ weakly stochastically dominates $\pi_{j}$ at $R_{i}$. The corresponding requirement of a correspondence $\Phi$ is as follows:

Sd no-envy: For each $R \in \mathcal{R}^{N}$ and each $\pi \in \Phi(R), \pi$ is sd envy-free at $R$.

The last requirement is an invariance of a solution. Let $a \in A$. Suppose that, keeping each other agent's preference fixed, the preference of an agent, say agent $i$, changes, but that his upper contour set at an object, say $a$, remains the same, and that so does his ranking over this set. Consider an assignment matrix selected for the resulting profile. Consider the set of objects that agent $i$ finds at least as desirable as $a$. We require that for agent $i$, the total probability of his receiving these objects should remain the same. For each pair $R_{0}, R_{0}^{\prime} \in \mathcal{R}$ and each $a \in A$, we write $R_{0}(a)=R_{0}^{\prime}(a)$ if $U\left(R_{0}, a\right)=U\left(R_{0}^{\prime}, a\right)$ and $\left.R_{0}\right|_{U\left(R_{0}, a\right)}=\left.R_{0}^{\prime}\right|_{U\left(R_{0}, a\right)}$. Similarly, for each pair $R_{0}, R_{0}^{\prime} \in \mathcal{R}$ and each $B \subseteq A$, we write $R_{0}(B)=R_{0}^{\prime}(B)$ if $U\left(R_{0}, B\right)=U\left(R_{0}^{\prime}, B\right)$ and $\left.R_{0}\right|_{U\left(R_{0}, B\right)}=\left.R_{0}^{\prime}\right|_{U\left(R_{0}, B\right)}$.

Limited invariance: For each $R \in \mathcal{R}^{N}$, each $i \in N$, each $a \in A$, and each $R_{i}^{\prime} \in \mathcal{R}$, if $R_{i}(a)=$ $R_{i}^{\prime}(a)$, then for each $\pi \in \Phi(R)$ and each $\pi^{\prime} \in \Phi\left(R_{i}^{\prime}, R_{-i}\right), \sum_{b \in U\left(R_{i}, a\right)} \pi_{i b}=\sum_{b \in U\left(R_{i}, a\right)} \pi_{i b}^{\prime}$.

Although solutions that we consider take agents' ordinal preferences as input, agents may have cardinal preferences as private information. For example, an agent may find his most preferred object(s) much more desirable than the remaining objects. Similarly, for some $t \in$ $\{1, \cdots,|A|\}$, an agent may find his $t$-most preferred objects much more desirable than the remaining objects. ${ }^{7}$ By imposing our invariance requirement, we prevent profitable manipulations

\footnotetext{
${ }^{7}$ Let us call the objects that are $t$-th ranked or above at the preference the " $t$-most preferred objects". Such cardinal preferences are common when agents have their own "lower bound"; agents rank objects that are below their lower bounds, but they do not take those objects seriously.
} 
by each agent who has these types of preferences. ${ }^{8}$ First, consider a possibility that an agent only counts his most preferred object(s), say $a$; suppose that his preference changes but his most preferred object remains the same; if the probability assigned to $a$ changes, say increases, then he has an incentive to misrepresent his preferences; thus, the probability assigned to $a$ should remain the same. Second, consider a possibility that an agent only counts his most preferred object(s) and the second most preferred object(s), say $a$ and $\{b, c\}$ (where the objects in the parenthesis are the objects that he finds equally desirable); suppose that his preference changes but his most preferred object and the second most preferred objects remain the same; by the first step, the probability assigned to $a$ should remain the same; if the total probability assigned to $\{b, c\}$ changes, say increases, then he has an incentive to misrepresent his preferences; thus, the total probability assigned to $\{b, c\}$ should also remain the same. Repeating this argument is exactly what limited invariance requires. ${ }^{9}$ For a different interpretation of the requirement, see Hashimoto et al. (2013).

\subsection{The Extended Serial Correspondence}

Katta and Sethuraman (2006) propose an adaptation of the serial rule to profiles of (possibly) weak preferences. As readers familiar with the difficulties generated by indifference might expect, their proposal is not a rule but a correspondence. However, we should note that for each economy, each agent is indifferent between any two of the assignment matrices selected by the correspondence. Thus, this correspondence is "essentially single-valued". Let us call this solution the extended serial correspondence (ES).

Let $R \in \mathcal{R}^{N}$. Let $A_{0}=A, X_{0}=\emptyset, \lambda_{0}^{*}=0$, and for each $i \in N, s_{0}(i)=0$. For each $m \in \mathbb{N}_{+}$,

Step $\boldsymbol{m}$. Let $\lambda_{m} \in\left[0,1-\sum_{s=1}^{m-1} \lambda_{s}^{*}\right]$. Let $A_{m} \equiv A_{m-1} \backslash X_{m-1}$. Let

$$
M^{*} \in \underset{M \subseteq N}{\operatorname{argmin}} \frac{\left|C h\left(R_{M}, A_{m}\right)\right|-\sum_{i \in M} s_{m-1}(i)}{|M|}
$$

and call such $M^{*}$ a "bottleneck set" at Step $m .{ }^{10}$ Let $\lambda_{m}^{*} \equiv \frac{\left|C h\left(R_{M^{*}}, A_{m}\right)\right|-\sum_{i \in M^{*}} s_{m-1}(i)}{\left|M^{*}\right|}$. Let $N_{m} \equiv \bigcup_{M^{*} \subseteq N}\left\{M^{*}: M^{*}\right.$ is a bottleneck set at Step $\left.m\right\}$ and $X_{m} \equiv \sum_{i \in N_{m}} C h\left(R_{i}, A_{m}\right)$. For

\footnotetext{
${ }^{8}$ An incentive requirement in Bogomolnaia and Moulin (2001) is defined independently of such cardinal preferences: no matter what his cardinal preferences are, each agent should not be sd better off by misreporting his (ordinal) preferences, given any other agents preferences. However, this requirement is so demanding that it is not compatible with sd efficiency and a basic fairness requirement called "equal treatment of equals" (Bogomolnaia and Moulin, 2001).

${ }^{9}$ From a social planner's view, $t$ is not known publicly and thus it makes sense to consider each $t \in\{1, \cdots,|A|\}$ as above. Here is an numerical example of these arguments. Consider a rule and let $R \in \mathcal{R}^{N}$ and $i \in N$. Let $R_{i}:\{a, b\} c d e$ and represent his cardinal preference by a function $u_{i}: A \rightarrow \mathbb{R}_{+}$such that $u_{i}(a)=u_{i}(b)=100$, $u_{i}(c)=\epsilon, u_{i}(d)=\epsilon^{2}$, and $u_{i}(e)=\epsilon^{3}$ where $\left.\epsilon \in\right] 0,1\left[\right.$. Let $R_{i}^{\prime} \in \mathcal{R}$ be such that $R_{i}(\{a, b\})=R_{i}^{\prime}(\{a, b\})$. As $\epsilon \rightarrow 0$, agent $i$ finds $\{a, b\}$ much more desirable than the other objects. To prevent this agent from benefiting by misrepresenting his preferences, the total probability assigned to $\{a, b\}$ should remain the same. We could also continue to the case with $R_{i}(c)=R_{i}^{\prime}(c)$ by setting $u_{i}(a)=u_{i}(b)=100, u_{i}(c)=99, u_{i}(d)=\epsilon$, and $u_{i}(e)=\epsilon^{2}$.

${ }^{10}$ For an alternative definition using max-flow graphs, see Katta and Sethuraman (2006).
} 
each $i \in N_{m}$, let $s_{m}(i)=0$ and assign the probability $\left(s_{m-1}(i)+\lambda_{m}^{*}\right)$ of objects in $C h\left(R_{i}, A_{m}\right)$. For each $i \notin N_{m}$, let $s_{m}(i) \equiv s_{m-1}(i)+\lambda_{m}^{*}$.

We say that at Step $m$, the (probability) supply of each object in $X_{m}$ reaches exhaustion, or simply, that $\boldsymbol{X}_{\boldsymbol{m}}$ reaches exhaustion. ${ }^{11}$ The algorithm terminates at Step $\bar{m}$ if either $A_{\bar{m}}=\emptyset$ or $\sum_{s=1}^{\bar{m}} \lambda_{s}^{*} \geq 1$. Note that $\bar{m} \leq|A|$. Here is an example of how the ES algorithm works.

Example. Let $A \equiv\{a, b, c\}, N \equiv\{1,2,3\}$, and $R \in \mathcal{R}^{N}$ be such that $R_{1}: a\{b, c\}, R_{2}: a c b$, and $R_{3}:\{a, b, c\}$ where the equally desirable objects are represented in the parentheses. The ES algorithm terminates in two steps:

Step 1. Let $\lambda_{1} \in[0,1]$ and $A_{1} \equiv\{a, b, c\}$. The bottleneck set at Step 1 is $M^{*}=\{1,2\}(=$ $N_{1}$ ) with $\lambda_{1}^{*}=\frac{1}{2}$ and $X_{1}=\{a\}$. For agents 1 and 2 , assign the probability $\frac{1}{2}$ of $a$ and set $s_{1}(1)=s_{1}(2) \equiv 0$. For agent 3 , set $s_{1}(3) \equiv \frac{1}{2}$.

Step 2. Let $\lambda_{2} \in\left[0, \frac{1}{2}\right]$ and $A_{2} \equiv\{b, c\}$. The bottleneck set at Step 2 is $M^{*}=\{1,2,3\}\left(=N_{2}\right)$ with $\lambda_{2}^{*}=\frac{1}{2}$ and $X_{2}=\{b, c\}$. For agent 1 , assign the probability of $\frac{1}{2}$ of $\{b, c\}$ in total; for agent 2 , assign the probability $\frac{1}{2}$ of $c$; and for agent 3 , assign the probability 1 of $\{b, c\}$. There are infinitely many ES assignment matrices. ${ }^{12}$

The ES correspondence is essentially single-valued: for each $R \in \mathcal{R}^{N}$, each pair $\pi, \pi^{\prime} \in$ $E S(R)$, each $i \in N$, and each $a \in A, \sum_{b \in U\left(R_{i}, a\right)} \pi_{i b}=\sum_{b \in U\left(R_{i}, a\right)} \pi_{i b}^{\prime}$. With a slight abuse of notation, let $\sum_{b \in U\left(R_{i}, a\right)} \pi_{i b} \equiv \sum_{b \in U\left(R_{i}, a\right)} E S_{i b}(R)$.

\section{Main result}

Our main result is a characterization of the ES correspondence (in welfare terms) by means of sd-efficiency, sd no-envy, and limited invariance. In particular, we show that the ES is the largest correspondence that satisfies these three properties. We emphasize that although ES is not single-valued, it is essentially single-valued. The following family of rules shows that the ES correspondence is not the only solution satisfying the three requirements.

Example. Let $N \times A \equiv\{(i, a): i \in N$ and $a \in A\}$ and let $\chi$ be a strict ordering defined over $N \times A$. Let $\Phi^{\chi}$ be the subcorrespondence of the ES correspondence that selects, for each economy, the assignment matrix that maximizes the probability of each agent receiving each object in a lexicographic way according to $\chi$. Let $R \in \mathcal{R}^{N}$ and $(i, a)$ be the first pair according to $\chi$. Let $\Pi_{0} \equiv\left\{\pi \in E S(R)\right.$ : for each $\left.\pi^{\prime} \in E S(R), \pi_{i a} \geq \pi_{i a}^{\prime}\right\}$. If $\left|\Pi_{0}\right|=1$, then $\Phi^{\chi}(R)=\Pi_{0}$. If $\left|\Pi_{0}\right|>1$, then consider the second pair according to $\chi$, say $(j, b)$. Let $\Pi_{1} \equiv\left\{\pi \in \Pi_{0}\right.$ : for each $\left.\pi^{\prime} \in \Pi_{0}, \pi_{j b} \geq \pi_{j b}^{\prime}\right\}$. If $\left|\Pi_{1}\right|=1$, then $\Phi^{\chi}(R)=\Pi_{1}$. Otherwise, continue until a single assignment matrix is obtained. It is easy to check that this rule satisfies the three requirements.

\footnotetext{
${ }^{11}$ We adopted this term because, along the ES algorithm, each object belongs to at most one bottleneck set: if an object is in the bottleneck set at a certain step, all of its probability is distributed to agents at the step.

${ }^{12}$ That is, there are infinitely many ways to assign probabilities of $b$ and $c$ to agents 1 and 3 in Step 2 . For a detailed discussion on the existence of such assignment matrices, see Katta and Sethuraman (2006).
} 
Here is our main result:

\section{Theorem 1.}

(i) ES satisfies sd-efficiency, sd no-envy, and limited invariance.

(ii) If a correspondence $\Phi$ satisfies sd-efficiency, sd no-envy, and limited invariance, then $\Phi \subseteq E S$.

Proof. (i) First, ES satisfies sd-efficiency and sd no-envy (Katta and Sethuraman, 2006). Next, we show that ES satisfies limited invariance. Let $R \in \mathcal{R}^{N}, i \in N$, and $a \in A$. Let $R_{i}^{\prime} \in \mathcal{R}$ be such that $R_{i}(a)=R_{i}^{\prime}(a)$ and let $R^{\prime} \equiv\left(R_{i}^{\prime}, R_{-i}\right)$. Let $s \in\{1, \cdots,|A|\}$ be the step of the ES algorithm at which $a$ reaches exhaustion. From the definition, it follows that the sequence $\left(N_{l}, X_{l}, \lambda_{l}^{*}\right)_{l=1}^{s}$ obtained from Step 1 to Step $s$ of the ES algorithm for $R$ is the same as that for $R^{\prime}$.

(ii) Let $\Phi$ be a correspondence satisfying sd-efficiency, sd no-envy, and limited invariance. Let $s \in\{0,1,2, \cdots,|A|-1\}$. By induction, suppose that for each $R \in \mathcal{R}^{N}$, each $\pi \in \Phi(R)$ coincides with an assignment matrix in $E S(R)$ on the objects that reach exhaustion before or at Step $s$ of the ES algorithm for $R .^{13}$ (denote this hypothesis by (*)) We prove that $\pi$ coincides with an assignment matrix in $E S(R)$ on the objects that reach exhaustion at Step $(s+1)$ of the ES algorithm for $R$. Then, an induction argument completes the proof.

Let $R \in \mathcal{R}^{N}$. Let $\bar{X} \equiv \bigcup_{l=1}^{s} X_{l}$ be the set of objects that reach exhaustion before or at Step $s$ of the ES algorithm for $R$ (if $s=0$, then let $\bar{X} \equiv \emptyset$ ). For each $i \in N$, let $C h(i) \equiv C h\left(R_{i}, A_{s+1}\right)$. Let $m \in \mathbb{N}_{+}$. Let $\mathcal{N} \equiv\left(N^{1}, N^{2}, \cdots, N^{m}\right)$ be a partition of $N$. For each $k \in\{1, \cdots, m\}$, let

- $N^{1, \cdots, k} \equiv \bigcup_{l=1}^{k} N^{l}$ and $N^{0} \equiv \emptyset$,

- $C h\left(N^{k}\right) \equiv\left[\bigcup_{i \in N^{k}} C h(i)\right] \backslash\left[\bigcup_{j \in N^{1, \cdots, k-1}} C h(j)\right]$ and $C h\left(N^{0}\right) \equiv \emptyset,{ }^{14}$

- $C h\left(N^{1, \cdots, k}\right) \equiv \bigcup_{l=1}^{k} C h\left(N^{l}\right)\left(=\bigcup_{i \in N^{1, \cdots, k}} C h(i)\right)$, and

- $G(k, R, \mathcal{N}) \equiv\left|C h\left(N^{1, \cdots, k}\right)\right|+\sum_{i \in N^{1, \cdots, k}} \sum_{a \in \bar{X}} E S_{i a}(R)$.

Note that $G(k, R, \mathcal{N})$ is the total probability of $\bar{X} \cup C h\left(N^{1, \cdots, k}\right)$ that can be assigned to the members of $N^{1, \cdots, k}$ under the induction hypothesis. Let $\tau(k, R, \mathcal{N}) \equiv \frac{G(k, R, \mathcal{N})}{\left|N^{1, \cdots, k}\right|}$. Here are useful observations that we exploit throughout the proof.

Lemma 1. Let $\mathcal{N} \equiv\left(N^{1}, \cdots, N^{m}\right)$ be a partition of $N$. Let $R^{\prime} \in \mathcal{R}^{N}$ be such that for each $i \in N, R_{i}(C h(i))=R_{i}^{\prime}(C h(i))$.

(i) For each $\bar{\pi} \in E S(R)$, each $\bar{\pi}^{\prime} \in E S\left(R^{\prime}\right)$, each $i \in N$, and each $a \in \bar{X}, \sum_{b \in I\left(R_{i}, a\right)} \bar{\pi}_{i b}=\sum_{b \in I\left(R_{i}^{\prime}, a\right)} \bar{\pi}_{i b}^{\prime}$.

(ii) For each $\pi \in \Phi\left(R^{\prime}\right)$, each $i \in N$, and each $a \in \bar{X} \backslash U\left(R_{i}^{\prime}, C h(i)\right), \pi_{i a}=0$.

(iii) For each $k \in\{1, \cdots, m\}, G(k, R, \mathcal{N})=G\left(k, R^{\prime}, \mathcal{N}\right)$.

Proof: It follows from the induction hypothesis and the definition of the ES correspondence.

\footnotetext{
${ }^{13}$ If $s=0$, then the induction hypothesis is vacuously true.

${ }^{14}$ Note that from the construction, for each pair of distinct $k, l \in\{1, \cdots, m\}, C h\left(N^{k}\right)$ and $C h\left(N^{l}\right)$ are disjoint.
} 
Now, among all possible partitions of $N$, we construct a partition $\mathcal{N}^{*} \equiv\left(N^{1}, N^{2}, \cdots, N^{m}\right)$ as follows: let $N^{1} \subseteq N$ be the largest solution of

$$
\min _{T \subseteq N} \frac{\left|\bigcup_{i \in T} C h(i)\right|+\sum_{i \in T} \sum_{o \in \bar{X}} E S_{i o}(R)}{|T|}
$$

and for each $k \in\{2, \cdots, m\}$, let $N^{1, \cdots, k}$ be the largest solution of the same problem with an additional constraint $N^{1, \cdots, k-1} \subsetneq T$. Then, we obtain a sequence $\left(N^{1}, N^{1,2}, \cdots, N^{1, \cdots, m}\right)$ such that $\emptyset \neq N^{1} \subsetneq N^{1,2} \subsetneq \ldots \subsetneq N^{1, \ldots, m}=N$. For each $k \in\{2, \cdots, m\}$, let $N^{k} \equiv N^{1, \ldots, k} \backslash$ $N^{1, \ldots, k-1} .15$ From the construction of $\mathcal{N}^{*}, \tau\left(k, R, \mathcal{N}^{*}\right)$ is strictly increasing in $k \in\{1, \cdots, m\}$. For each $k \in\{1, \cdots, m\}$, we also have $C h\left(N^{k}\right) \neq \emptyset .{ }^{16}$

Note that $\mathcal{N}^{*}$ is determined by the preference profile and that $\mathcal{N}^{*}$ remains the same if $R$ changes to any preference profile $R^{\prime}$ as described in Lemma 1. All the profiles in the proof below is as described in Lemma 1. By Lemma 1(iii), we can omit $R^{\prime}$ and $\mathcal{N}^{*}$ as arguments of $\tau\left(k, R^{\prime}, \mathcal{N}^{*}\right)$.

For each $l \in\{1, \cdots, m\}$ and each $i \in N^{l}$, let $\bar{R}_{i} \in \mathcal{R}$ be the preference at which agent $i$ ranks the objects as follows:

- he ranks the objects from the most preferred object down to $C h(i)$ in the same way as he does at $R_{i}$,

- he finds the objects in $\left[\bar{X} \cup C h\left(N^{l}\right)\right] \backslash U\left(R_{i}, C h(i)\right)$ indifferent and ranks them just below $C h(i)$,

- he finds the objects in $C h\left(N^{1}\right) \backslash C h(i)$ indifferent and ranks them just below $\left[\bar{X} \cup C h\left(N^{l}\right)\right] \backslash$ $U\left(R_{i}, C h(i)\right),{ }^{17}$

- for each $k \in\{2, \cdots, l-1\}$, he finds the objects in $C h\left(N^{k}\right) \backslash C h(i)$ indifferent and ranks them just below $C h\left(N^{k-1}\right) \backslash C h(i)$,

- he finds the objects in $C h\left(N^{l+1}\right)$ indifferent and ranks them just below $C h\left(N^{l-1}\right) \backslash C h(i)$,

\footnotetext{
${ }^{15}$ The construction itself establishes the existence of such a partition. We thank the anonymous referee for suggesting this representation of $\mathcal{N}^{*}$.

${ }^{16}$ The proof is as follows. First, it is trivial that $C h\left(N^{1}\right) \neq \emptyset$. Next, suppose, by contradiction, that there is $k \in\{2, \cdots, m\}$ such that $C h\left(N^{k}\right)=\emptyset$. Then, $C h\left(N^{1, \cdots, k}\right)=C h\left(N^{1, \cdots, k-1}\right)$. From the definition of $\tau(\cdot)$,

$$
\begin{aligned}
\tau\left(k, R, \mathcal{N}^{*}\right)\left|N^{1, \cdots, k}\right| & =\left|C h\left(N^{1, \cdots, k}\right)\right|+\sum_{i \in N^{1, \cdots, k}} \sum_{o \in \bar{X}} E S_{i o}(R) \\
& =\left|C h\left(N^{1, \cdots, k-1}\right)\right|+\sum_{i \in N^{1, \cdots, k-1}} \sum_{o \in \bar{X}} E S_{i o}(R)+\sum_{i \in N^{k}} \sum_{o \in \bar{X}} E S_{i o}(R) \\
& =\tau\left(k-1, R, \mathcal{N}^{*}\right)\left|N^{1, \cdots, k-1}\right|+\sum_{i \in N^{k}} \sum_{o \in \bar{X}} E S_{i o}(R) .
\end{aligned}
$$
}

Rewriting LHS into $\tau\left(k, R, \mathcal{N}^{*}\right)\left(\left|N^{1, \cdots, k-1}\right|+\left|N^{k}\right|\right)$ and rearranging the equation, we obtain

$$
\begin{aligned}
\left(\tau\left(k, R, \mathcal{N}^{*}\right)-\tau\left(k-1, R, \mathcal{N}^{*}\right)\right)\left|N^{1, \cdots, k-1}\right| & =\sum_{i \in N^{k}} \sum_{o \in \bar{X}} E S_{i o}(R)-\tau\left(k, R, \mathcal{N}^{*}\right)\left|N^{k}\right| \\
& =\sum_{i \in N^{k}}\left(\sum_{o \in \bar{X}} E S_{i o}(R)-\tau\left(k, R, \mathcal{N}^{*}\right)\right) .
\end{aligned}
$$

Since for each $i \in N^{k}, \sum_{o \in \bar{X}} E S_{i o}(R)<\tau\left(1, R, \mathcal{N}^{*}\right)<\tau\left(k, R, \mathcal{N}^{*}\right)$, and thus LHS $<0$, contradicting that $\tau\left(k, R, \mathcal{N}^{*}\right)$ is strictly increasing in $k$.

${ }^{17}$ From the definition of $C h\left(N^{1}\right)$ and $C h\left(N^{l}\right)$, it follows that $C h\left(N^{1}\right) \backslash C h(i)$ and $\bar{X} \cup C h\left(N^{l}\right)$ are disjoint. 
- for each $k \in\{l+2, \cdots, m\}$, he finds the objects in $C h\left(N^{k}\right)$ indifferent and ranks them just below $C h\left(N^{k-1}\right)$, and

- he finds the remaining objects indifferent and ranks them below $C h\left(N^{m}\right)$.

Let $R^{l} \in \mathcal{R}^{N}$ be the preference profile $\left(\left(\bar{R}_{\gamma}\right)_{\gamma \in N^{1, \cdots, l}},\left(R_{\gamma}\right)_{\gamma \in N \backslash N^{1, \cdots, l}}\right)$ and $R^{0} \equiv R$. Let $\overline{\mathcal{R}}^{N}(l) \equiv$ $\left\{R^{\prime} \in \mathcal{R}^{N}\right.$ : for each $i \in N^{l}, R_{i}^{\prime}(C h(i))=R_{i}^{l}(C h(i))$ and for each $\left.i \notin N^{l}, R_{i}^{\prime}=R_{i}^{l}\right\}$. Note that $R^{l-1}, R^{l} \in \overline{\mathcal{R}}^{N}(l)$.

Remark 1. For each $l, k \in\{1, \cdots, m\}$ with $k \leq l$, (i) $C h\left(N^{k}\right)$ is the set of objects that reach exhaustion at Step $(s+k)$ under the ES algorithm at each $\bar{R}^{l} \in \overline{\mathcal{R}}^{N}(l)$ (that is, $C h\left(N^{k}\right)=X_{s+k}$ ) and (ii) $\sum_{t=1}^{s+k} \lambda_{t}^{*}=\tau(k)$. Note also that $C h\left(N^{1}\right)$ coincides with the set of objects that reach exhaustion at Step $(s+1)$ under the ES algorithm at $R^{0}(=R)$. These statements follow from the definition of the ES correspondence and the construction of $\mathcal{N}^{*}$.

Lemma 2. Let $t \in\{0, \cdots, m-1\}$ and $\bar{R}^{t+1} \in \overline{\mathcal{R}}^{N}(t+1)$. Let $\pi \in \Pi$ be an sd envy-free matrix at $\bar{R}^{t+1}$. For each $i \in N^{1, \cdots, t+1}$ and each $a \in C h\left(N^{1, \cdots, t+1}\right), \sum_{o \in U\left(\bar{R}^{t+1}, a\right)} E S_{i o}\left(\bar{R}^{t+1}\right) \geq$ $\sum_{o \in U\left(\bar{R}^{t+1}, a\right)} \pi_{i o}$

Proof. Suppose, by contradiction, that there are an sd envy-free matrix $\pi \in \Pi, i \in$ $N^{1, \cdots, t+1}$, and $a \in C h\left(N^{1, \cdots, t+1}\right)$ such that $\sum_{o \in U\left(\bar{R}_{i}^{t+1}, a\right)} \pi_{i o}>\sum_{o \in U\left(\bar{R}_{i}^{t+1}, a\right)} E S_{i o}\left(\bar{R}^{t+1}\right)$. Let $l, k \in\{1, \cdots, t+1\}$ be such that $i \in N^{l}$ and $a \in C h\left(N^{k}\right)$. There are two cases.

Case 1. $\boldsymbol{l}<\boldsymbol{k}$ : From the way we construct $\bar{R}^{t+1}$ and Remark $1, \sum_{o \in U\left(\bar{R}_{i}^{t+1}, a\right)} E S_{i o}\left(\bar{R}^{t+1}\right)=$ $\tau(k)$. It also follows that $U\left(\bar{R}_{i}^{t+1}, a\right)=\bar{X} \cup C h\left(N^{1, \cdots, k}\right) .{ }^{18}$ Altogether,

$$
\tau(k)=\sum_{o \in U\left(\bar{R}_{i}^{t+1}, a\right)} E S_{i o}\left(\bar{R}^{t+1}\right)<\sum_{o \in U\left(\bar{R}_{i}^{t+1}, a\right)} \pi_{i o}=\sum_{o \in \bar{X} \cup C h\left(N^{1, \cdots, k}\right)} \pi_{i o} .
$$

By sd no-envy, for each $j \in N^{1, \cdots, k}, \sum_{o \in \bar{X} \cup C h\left(N^{1, \cdots, k}\right)} \pi_{j o} \geq \sum_{o \in \bar{X} \cup C h\left(N^{1, \cdots, k}\right)} \pi_{i o}>\tau(k)$. Summing over $N^{1, \cdots, k}$, we have

$$
\sum_{j \in N^{1, \cdots, k}} \sum_{o \in \bar{X} \cup C h\left(N^{1, \cdots, k}\right)} \pi_{j o}>\tau(k)\left|N^{1, \cdots, k}\right|=\sum_{j \in N^{1, \cdots, k}} \sum_{o \in \bar{X}} E S_{j o}\left(\bar{R}^{t+1}\right)+\left|C h\left(N^{1, \cdots, k}\right)\right|,
$$

where the equality comes from the definition of $\tau(\cdot)$. By ( $*)$ and Lemma $1(\mathrm{i}), \sum_{j \in N^{1, \cdots, k}} \sum_{o \in \bar{X}} \pi_{j o}=$ $\sum_{j \in N^{1, \cdots, k}} \sum_{o \in \bar{X}} E S_{j o}\left(\bar{R}^{t+1}\right)$. Thus, $\sum_{j \in N^{1, \cdots, k}} \sum_{o \in C h\left(N^{1, \cdots, k}\right)} \pi_{j o}>\left|C h\left(N^{1, \cdots, k}\right)\right|$, a violation of feasibility.

Case 2. $\boldsymbol{l} \geq \boldsymbol{k}$ : From the way we construct $\bar{R}^{t+1}$ and Remark $1, \sum_{o \in U\left(\bar{R}_{i}^{t+1}, a\right)} E S_{i o}\left(\bar{R}^{t+1}\right)=$ $\tau(l)$. It also follows that $U\left(\bar{R}_{i}^{t+1}, a\right) \subseteq \bar{X} \cup C h\left(N^{1, \cdots, l}\right) .{ }^{19}$ Altogether,

$$
\tau(l)=\sum_{o \in U\left(\bar{R}_{i}^{t+1}, a\right)} E S_{i o}\left(\bar{R}^{t+1}\right)<\sum_{o \in U\left(\bar{R}_{i}^{t+1}, a\right)} \pi_{i o} \leq \sum_{o \in \bar{X} \cup C h\left(N^{1, \cdots, l}\right)} \pi_{i o} .
$$

\footnotetext{
${ }^{18}$ Recall that $\bar{R}_{i}^{t+1}$ is constructed as follows: $\left[\bar{X} \cup C h(i) \cup C h\left(N^{l}\right)\right] \quad \bar{P}_{i}^{t+1} \quad\left[C h\left(N^{1}\right) \backslash C h(i)\right] \quad \bar{P}_{i}^{t+1} \quad \ldots$ $\bar{P}_{i}^{t+1}\left[C h\left(N^{l-1}\right) \backslash C h(i)\right] \bar{P}_{i}^{t+1}\left[C h\left(N^{l+1}\right)\right] \bar{P}_{i}^{t+1} \ldots \bar{P}_{i}^{t+1}\left[C h\left(N^{k}\right)\right]$. Note that $a \in C h\left(N^{k}\right)$.

${ }^{19}$ Recall that $\bar{R}_{i}^{t+1}$ is constructed as follows: $\left[\bar{X} \cup C h(i) \cup C h\left(N^{l}\right)\right] \quad \bar{P}_{i}^{t+1} \quad\left[C h\left(N^{1}\right) \backslash C h(i)\right] \quad \bar{P}_{i}^{t+1} \quad \ldots$ $\bar{P}_{i}^{t+1}\left[C h\left(N^{k}\right) \backslash C h(i)\right] \bar{P}_{i}^{t+1} \ldots \bar{P}_{i}^{t+1}\left[C h\left(N^{l-1}\right) \backslash C h(i)\right]$. Note that either $a \in C h(i)$ or $a \in C h\left(N^{k}\right) \backslash C h(i)$.
} 
By sd no-envy, for each $j \in N^{1, \cdots, l}, \sum_{o \in \bar{X} \cup C h\left(N^{1, \cdots, l}\right)} \pi_{j o} \geq \sum_{o \in \bar{X} \cup C h\left(N^{1, \cdots, l}\right)} \pi_{i o}>\tau(l)$. By the similar argument as in Case 1, we obtain a violation of feasibility.

Lemma 3. Let $t \in\{0, \cdots, m-1\}$ and $\pi \in \Phi\left(R^{t+1}\right)$. Suppose that for each $i \in N^{1, \cdots, t+1}$, $\sum_{o \in \bar{X} \cup C h\left(N^{1, \cdots, t+1}\right)} \pi_{i o}=\tau(t+1)<1$. Then, $\sum_{i \in N^{1, \cdots, t+1}} \sum_{o \in C h\left(N^{1, \cdots, t+1}\right)} \pi_{i o}=\left|C h\left(N^{1, \cdots, t+1}\right)\right|$.

Proof. Summing over the equation in the hypothesis of Lemma 3 over $N^{1, \cdots, t+1}$ and then rearranging the equation,

$$
\sum_{i \in N^{1, \cdots, t+1}} \sum_{o \in C h\left(N^{1, \cdots, t+1}\right)} \pi_{i o}=\tau(t+1)\left|N^{1, \cdots, t+1}\right|-\sum_{i \in N^{1, \cdots, t+1}} \sum_{o \in \bar{X}} \pi_{i o} .
$$

From the definition of $\tau(\cdot), \tau(t+1)\left|N^{1, \cdots, t+1}\right|=\left|C h\left(N^{1, \cdots, t+1}\right)\right|+\sum_{i \in N^{1, \cdots, t+1}} \sum_{o \in \bar{X}} E S_{i o}\left(R^{t+1}\right)$. By (*) and Lemma 1(i), $\sum_{i \in N^{1, \cdots, t+1}} \sum_{o \in \bar{X}} \pi_{i o}=\sum_{i \in N^{1, \cdots, t+1}} \sum_{o \in \bar{X}} E S_{i o}\left(R^{t+1}\right)$. Altogether, $\sum_{i \in N^{1, \cdots, t+1}} \sum_{o \in C h\left(N^{1, \cdots, t+1}\right)} \pi_{i o}=\left|C h\left(N^{1, \cdots, t+1}\right)\right|$, completing the proof.

Lemma 4. Let $t \in\{0, \cdots, m-1\}$ and $\pi \in \varphi\left(R^{t+1}\right)$. Suppose that for each $i \in N^{1, \cdots, t+1}$, $\sum_{o \in \bar{X} \cup C h\left(N^{1, \cdots, t+1}\right)} \pi_{i o}=\tau(t+1)<1$. Then, $\pi$ coincides with an ES assignment for $R^{t+1}$ on $\operatorname{Ch}\left(N^{1, \cdots, t+1}\right)$.

Proof. Suppose, by contradiction, that $\pi$ does not coincide with any ES assignment matrix for $R^{t+1}$ on $C h\left(N^{1, \cdots, t+1}\right)$. Let $\bar{\pi} \in \Pi$ be a matrix such that $\bar{\pi}$ coincides with an ES assignment matrix for $R^{t+1}$ on $C h\left(N^{1, \cdots, t+1}\right)$ and $\bar{\pi}$ coincides with $\pi$ on $A \backslash C h\left(N^{1, \cdots, t+1}\right)$. By (*), Lemma 1 (i), and Lemma $3, \bar{\pi}$ is feasible. By Lemma $2, \bar{\pi}$ stochastically dominates $\pi$ at $R^{t+1}$, in violation of sd-efficiency.

Corollary 1. Let $t \in\{1, \cdots, m-1\}$ and $\pi \in \Phi\left(R^{t+1}\right)$. Suppose that for each $i \in N^{1, \cdots, t+1}$, $\sum_{o \in \bar{X} \cup C h\left(N^{1, \cdots, t+1}\right)} \pi_{i o}=\tau(t+1)<1$. Let $l \in\{1, \cdots, t\}$. Then,

(i) For each $i \in N^{1, \cdots, l}, \sum_{o \in \bar{X} \cup C h\left(N^{1, \cdots, l}\right)} \pi_{i o}=\tau(l)$.

(ii) For each $i \in N^{l}$ and each $o \in C h\left(N^{l}\right) \backslash C h(i), \pi_{i o}=0$.

(iii) For each $i \in N^{l+1}$ and each $o \in C h\left(N^{1, \cdots, l}\right), \pi_{i o}=0$.

Proof. It follows from Lemma 4.

Lemma 5. Let $t \in\{1, \cdots, m\}$ and $\bar{R} \in \overline{\mathcal{R}}^{N}(t)$. Suppose that for each $\pi \in \Phi\left(R^{t}\right)$ and each $i \in N^{1, \cdots, t}, \sum_{o \in \bar{X} \cup C h\left(N^{1, \cdots, t}\right)} \pi_{i o}=\tau(t)<1$. Then, each $\bar{\pi} \in \Phi(\bar{R})$ is such that for each $i \in N^{t}$ and each $o \in C h\left(N^{t}\right) \backslash C h(i), \bar{\pi}_{i o}=0$.

Proof: Let $\pi \in \Phi\left(R^{t}\right)$ and $\bar{R} \in \overline{\mathcal{R}}^{N}(t)$. We start from $R^{t}$ and change the preference of each agent in $N^{t}$, one agent at a time, until we reach $\bar{R}$. By Corollary 1(ii), for each $i \in N^{t}$, and each $o \in C h\left(N^{t}\right) \backslash C h(i), \pi_{i o}=0$.

Step 1. Let $i \in N^{t}$. We change agent $i$ 's preference from $R_{i}^{t}$ to $\bar{R}_{i}$. By limited invariance, for each $\bar{\pi}^{1} \in \Phi\left(\bar{R}_{i}, R_{N \backslash\{i\}}^{t}\right), \sum_{o \in \bar{X} \cup C h(i)} \pi_{i o}=\sum_{o \in \bar{X} \cup C h(i)} \bar{\pi}_{i o}^{1}(=\tau(t))$. By sd no-envy, for each $j \in$ 
$N^{1, \cdots, t}, \sum_{o \in \bar{X} \cup C h\left(N^{1, \cdots, t}\right)} \bar{\pi}_{j o}^{1} \geq \sum_{o \in \bar{X} \cup C h(i)} \bar{\pi}_{i o}^{1}(=\tau(t))$, and by feasibility, $\sum_{o \in \bar{X} \cup C h\left(N^{1, \cdots, t}\right)} \bar{\pi}_{j o}^{1}$ $=\tau(t)$. Summing over $N^{1, \cdots, t}$ and applying (*) and Lemma 1(i),

$$
\sum_{j \in N^{1, \cdots, t}} \sum_{o \in C h\left(N^{1, \cdots, t}\right)} \bar{\pi}_{j o}^{1}=\left|C h\left(N^{1, \cdots, t}\right)\right| .
$$

Suppose, by contradiction, that there are $\pi^{1} \in \Phi\left(\bar{R}_{i}, R_{N \backslash\{i\}}^{t}\right), k \in N^{t}$, and $o \in C h\left(N^{t}\right) \backslash$ $C h(k)$ such that $\pi_{k o}^{1}>0$. Then, $\pi^{1}$ does not coincide with any ES assignment for $\left(\bar{R}_{i}, R_{N \backslash\{i\}}^{t}\right)$ on $C h\left(N^{t}\right)$. Therefore, $\pi^{1} \notin E S\left(\bar{R}_{i}, R_{N \backslash\{i\}}^{t}\right)$. Since $\left(\bar{R}_{i}, R_{N \backslash\{i\}}^{t}\right) \in \overline{\mathcal{R}}^{N}(t)$, by Lemma 2, each ES matrix for $\left(\bar{R}_{i}, R_{N \backslash\{i\}}^{t}\right)$ stochastically dominates $\pi^{1}$ at $\left(\bar{R}_{i}, R_{N \backslash\{i\}}^{t}\right)$, in violation of sd-efficiency.

Step 2. Let $j \in N^{t} \backslash\{i\}$. We change agent $j$ 's preference from $R_{j}^{t}$ to $\bar{R}_{j}$. By limited invariance, for each $\bar{\pi}^{2} \in \Phi\left(\bar{R}_{i}, \bar{R}_{j}, R_{N \backslash\{i, j\}}^{t}\right), \sum_{o \in \bar{X} \cup C h(j)} \bar{\pi}_{j o}^{1}=\sum_{o \in \bar{X} \cup C h(j)} \bar{\pi}_{j o}^{2}(=\tau(t))$. For each $k \in N^{1, \cdots, t} \backslash\{i\}$, it follows from $s d$ no-envy that $\sum_{o \in \bar{X} \cup C h\left(N^{1, \cdots, t}\right)} \bar{\pi}_{k o}^{2} \geq \tau(t)\left(=\sum_{o \in \bar{X} \cup C h(j)} \bar{\pi}_{j o}^{2}\right)$. For agent $i$, we show that $\sum_{o \in \bar{X} \cup C h(i)} \bar{\pi}_{i o}^{2}=\tau(t)$. Note that, by the argument in Step 1, for each $\hat{\pi} \in \Phi\left(\bar{R}_{j}, R_{N \backslash\{j\}}^{t}\right)$, we have $\sum_{o \in \bar{X} \cup C h(i)} \hat{\pi}_{i o}=\tau(t)$. By limited invariance, $\sum_{o \in \bar{X} \cup C h(i)} \hat{\pi}_{i o}=$ $\sum_{o \in \bar{X} \cup C h(i)} \bar{\pi}_{i o}^{2}$. Altogether, by feasibility, for each $k \in N^{1, \cdots, t}, \sum_{o \in \bar{X} \cup C h\left(N^{1, \cdots, t}\right)} \bar{\pi}_{k o}^{2}=\tau(t)$. By the same argument in Step 1, for each $k \in N^{t}$ and each $o \in C h\left(N^{t}\right) \backslash C h(k), \bar{\pi}_{k o}^{2}=0$.

We repeat this process until we reach $\bar{R}$ : for each $\bar{\pi} \in \Phi(\bar{R})$, each $i \in N^{t}$, and each $o \in$ $C h\left(N^{t}\right) \backslash C h(i)$, we have $\bar{\pi}_{i o}=0$.

Lemma 6. Let $t \in\{0, \cdots, m-1\}$ and $\pi \in \Phi\left(R^{t}\right)$. Suppose that for each $\pi^{\prime} \in \Phi\left(R^{t+1}\right)$ and each $i \in N^{1, \cdots, t+1}, \sum_{o \in \bar{X} \cup C h\left(N^{1, \cdots, t+1}\right)} \pi_{i o}^{\prime}=\tau(t+1)<1$. Then, for each $\bar{\pi} \in \Phi\left(R^{t+1}\right)$ and each $i \in N^{t+1}, \sum_{o \in \bar{X} \cup C h\left(N^{t+1}\right)} \bar{\pi}_{i o} \leq \sum_{o \in \bar{X} \cup C h\left(N^{t+1}\right)} \pi_{i o}$.

Proof: Let $\pi \in \Phi\left(R^{t}\right)$. We start from $R^{t}$ and change the preferences of $N^{t+1}$, one agent at a time. Let $i \in N^{t+1}$. We change agent $i$ 's preference from $R_{i}^{t}$ to $R_{i}^{t+1}$. Then, for each $\pi^{1} \in$ $\Phi\left(R_{i}^{t+1}, R_{N \backslash\{i\}}^{t}\right)$ and each $k \in N^{t+1}$,

$$
\begin{aligned}
\sum_{o \in \bar{X} \cup C h\left(N^{t+1}\right)} \pi_{i o} & =\sum_{o \in \bar{X} \cup C h(i)} \pi_{i o}=\sum_{o \in \bar{X} \cup C h(i)} \pi_{i o}^{1} \\
& =\sum_{o \in \bar{X} \cup C h\left(N^{t+1}\right)} \pi_{i o}^{1} \geq \sum_{o \in \bar{X} \cup C h\left(N^{t+1}\right)} \pi_{k o}^{1}
\end{aligned}
$$

where the first and third equalities come from the fact that $R^{t},\left(R_{i}^{t+1}, R_{N \backslash\{i\}}^{t}\right) \in \overline{\mathcal{R}}^{N}(t+1)$ and Lemma 5 , the second equality from limited invariance, and the inequality from sd no-envy.

Next, let $j \in N^{t+1} \backslash\{i\}$. We change agent $j$ 's preference from $R_{j}^{t}$ to $R_{j}^{t+1}$. Then, for each $\pi^{2} \in \Phi\left(R_{i}^{t+1}, R_{j}^{t+1}, R_{N \backslash\{i, j\}}^{t}\right)$ and each $k \in N^{t+1}$,

$$
\begin{aligned}
\sum_{o \in \bar{X} \cup C h\left(N^{t+1}\right)} \pi_{j o}^{1} & =\sum_{o \in \bar{X} \cup C h(j)} \pi_{j o}^{1}=\sum_{o \in \bar{X} \cup C h(j)} \pi_{j o}^{2} \\
& =\sum_{o \in \bar{X} \cup C h\left(N^{t+1}\right)} \pi_{j o}^{2}=\sum_{o \in \bar{X} \cup C h\left(N^{t+1}\right)} \pi_{i o}^{2} \geq \sum_{o \in \bar{X} \cup C h\left(N^{t+1}\right)} \pi_{k o}^{2},
\end{aligned}
$$

where the first and third equalities come from the fact that $\left(R_{i}^{t+1}, R_{N \backslash\{i\}}^{t}\right),\left(R_{i}^{t+1}, R_{j}^{t+1}, R_{N \backslash\{i\}}^{t}\right) \in$ $\overline{\mathcal{R}}^{N}(t+1)$ and Lemma 5 , the second equality from limited invariance, and the fourth equality and the last inequality from sd no-envy. Altogether, $\sum_{o \in \bar{X} \cup C h\left(N^{t+1}\right)} \pi_{i o}^{1} \geq \sum_{o \in \bar{X} \cup C h\left(N^{t+1}\right)} \pi_{i o}^{2}$. 
We repeat this process, one agent at a time, until we reach $R^{t+1}$ : for each $\bar{\pi} \equiv \Phi\left(R^{t+1}\right)$, $\sum_{o \in \bar{X} \cup C h\left(N^{t+1}\right)} \pi_{i o} \geq \sum_{o \in \bar{X} \cup C h\left(N^{t+1}\right)} \bar{\pi}_{i o}$.

Now, we conclude the proof of the theorem. Let $\pi^{0} \in \Phi(R)$. By Remark 1, we are done if we prove that $\pi^{0}$ coincides with an assignment matrix in $E S(R)$ on $\bar{X} \cup C h\left(N^{1}\right)$. Suppose otherwise. Then, there is $k \in N^{1}$ such that $\sum_{o \in C h\left(N^{1}\right)} \pi_{k o}^{0}<\sum_{o \in C h\left(N^{1}\right)} E S_{k o}(R),{ }^{20}$ which implies $\sum_{o \in \bar{X} \cup C h\left(N^{1}\right)} \pi_{k o}^{0}<\tau(1)=\sum_{o \in \bar{X} \cup C h\left(N^{1}\right)} E S_{k o}(R)$ (denote this statement by $(\ddagger)$ ). We show that there is $\pi^{1} \in \Phi\left(R^{1}\right)$ such that for each $i \in N^{1}, \sum_{o \in \bar{X} \cup C h\left(N^{1}\right)} \pi_{i o}^{1}<\tau(1)<1$. First, if $\tau(1)=1$, then by Lemma 2 and the fact that $\pi^{0} \notin E S(R)$, each ES matrix stochastically dominates $\pi^{0}$ at $R$, in violation of sd-efficiency. Second, suppose that, for each $\pi^{1} \in \Phi\left(R^{1}\right)$, there is $i \in N^{1}$ such that $\sum_{o \in \bar{X} \cup C h\left(N^{1}\right)} \pi_{i o}^{1} \geq \tau(1)$. By sd no-envy, for each $k \in N^{1}, \sum_{o \in \bar{X} \cup C h\left(N^{1}\right)} \pi_{k o}^{1} \geq \tau(1)$, which implies, by feasibility, $\sum_{o \in \bar{X} \cup C h\left(N^{1}\right)} \pi_{k o}^{1}=\tau(1)$. Since $R, R^{1} \in \overline{\mathcal{R}}^{N}(1)$, Lemma 6 implies that, for each $\pi^{1} \in \Phi\left(R^{1}\right)$ and each $k \in N^{1}, \sum_{o \in \bar{X} \cup C h\left(N^{1}\right)} \pi_{k o}^{1}(=\tau(1)) \leq \sum_{o \in \bar{X} \cup C h\left(N^{1}\right)} \pi_{k o}^{0}$. By feasibility, again, the equality should hold, contradicting $(\ddagger)$.

Now, as an induction hypothesis, suppose that for each $t \in\{1, \cdots, m-1\}$, there is $\pi^{t} \in$ $\Phi\left(R^{t}\right)$ such that for each $i \in N^{1, \cdots, t}, \sum_{o \in \bar{X} \cup C h\left(N^{1, \cdots, t}\right)} \pi_{i o}^{t}<\tau(t)<1$. We prove that there is $\pi^{t+1} \in \Phi\left(R^{t+1}\right)$ such that for each $i \in N^{1, \cdots, t+1}, \sum_{o \in \bar{X} \cup C h\left(N^{1, \cdots, t+1}\right)} \pi_{i o}^{t+1}<\tau(t+1)<1$.

Claim 1. $\tau(t+1)<1$. Suppose, by contradiction, that $\tau(t+1)=1$. Then, $\pi^{t} \notin E S\left(R^{t}\right)$, and by Lemma 2, each ES matrix for $R^{t}$ stochastically dominates $\pi^{t}$ at $R^{t}$, in violation of sd-efficiency.

Claim 2. There is $\pi^{t+1} \in \Phi\left(R^{t+1}\right)$ such that for each $i \in N^{1, \cdots, t+1}, \sum_{o \in \bar{X}} \cup C h\left(N^{1, \cdots, t+1}\right) \pi_{i o}^{t+1}<$ $\tau(t+1)$. Suppose, by contradiction, that for each $\pi^{\prime} \in \Phi\left(R^{t+1}\right)$, there is $k \in N^{1, \cdots, t+1}$ such that $\sum_{o \in \bar{X} \cup C h\left(N^{1, \cdots, t+1}\right)} \pi_{k o}^{\prime}=\tau(t+1) .{ }^{21}$ By sd no-envy, for each $i \in N^{1, \cdots, t+1}$,

$$
\sum_{o \in \bar{X} \cup C h\left(N^{1, \cdots, t+1}\right)} \pi_{i o}^{\prime}=\sum_{o \in \bar{X} \cup C h\left(N^{1, \cdots, t+1}\right)} \pi_{k o}^{\prime}=\tau(t+1)(<1) .
$$

Now, we apply Lemma 6: for each $\pi^{t+1} \in \Phi\left(R^{t+1}\right)$ and each $j \in N^{t+1}, \sum_{o \in \bar{X} \cup C h\left(N^{t+1}\right)} \pi_{j o}^{t+1} \leq$ $\sum_{o \in \bar{X} \cup C h\left(N^{t+1}\right)} \pi_{j o}^{t}$. By (*) and Lemma 1(i), $\sum_{o \in C h\left(N^{t+1}\right)} \pi_{j o}^{t+1} \leq \sum_{o \in C h\left(N^{t+1}\right)} \pi_{j o}^{t}$. Summing the inequality over $N^{t+1}$, adding $\left|C h\left(N^{t+1}\right)\right|$ both sides, and then by rearranging the inequality, we have

$$
\begin{aligned}
\left|C h\left(N^{t+1}\right)\right|-\sum_{i \in N^{t+1}} \sum_{o \in C h\left(N^{t+1}\right)} \pi_{i o}^{t+1} & \geq\left|C h\left(N^{t+1}\right)\right|-\sum_{i \in N^{t+1}} \sum_{o \in C h\left(N^{t+1}\right)} \pi_{i o}^{t} \\
& \geq \sum_{i \in N^{1, \cdots, t}} \sum_{o \in C h\left(N^{t+1}\right)} \pi_{i o}^{t}
\end{aligned}
$$

where the last inquality comes from feasibility. Then, by $(\S)$ and Lemma 3, LHS is equal to $\sum_{i \in N^{1, \cdots, t}} \sum_{o \in C h\left(N^{t+1}\right)} \pi_{i o}^{t+1}$. Note that, by sd no-envy, for each $i, j \in N^{1, \cdots, t}$,

$$
\sum_{o \in C h\left(N^{t+1}\right)} \pi_{i o}^{t}=\sum_{o \in C h\left(N^{t+1}\right)} \pi_{j o}^{t} \text { and } \sum_{o \in C h\left(N^{t+1}\right)} \pi_{i o}^{t+1}=\sum_{o \in C h\left(N^{t+1}\right)} \pi_{j o}^{t+1} .
$$

\footnotetext{
${ }^{20}$ This comes from the following property of the ES correspondence: for each $R \in \mathcal{R}^{N}$ and each $\bar{\pi} \in E S(R)$, $E S(R)=\left\{\pi \in \Pi\right.$ : for each $t \in \mathbb{N}_{+}$, each $i \in N_{t}$, and each $\left.a \in X_{t}, \sum_{o \in U\left(R_{i}, a\right)} \pi_{i o}=\sum_{o \in U\left(R_{i}, a\right)} \bar{\pi}_{i o}\right\}$.

${ }^{21}$ If $\sum_{o \in \bar{X} \cup C h\left(N^{1, \cdots, t+1}\right)} \pi_{k o}^{\prime}>\tau(t+1)$, by sd no-envy, we obtain a violation of feasibility.
} 
Thus, for each $i \in N^{1, \cdots, t}, \sum_{o \in C h\left(N^{t+1}\right)} \pi_{i o}^{t+1} \geq \sum_{o \in C h\left(N^{t+1}\right)} \pi_{i o}^{t}$. From the induction hypothesis, for each $i \in N^{1, \cdots, t}$,

$$
\sum_{o \in \bar{X} \cup C h\left(N^{1, \cdots, t}\right)} \pi_{i o}^{t}<\tau(t)=\sum_{o \in \bar{X} \cup C h\left(N^{1, \cdots, t}\right)} \pi_{i o}^{t+1},
$$

where the equality comes from $(\S)$ and Corollary 1(i). Adding $\sum_{o \in C h\left(N^{t+1}\right)} \pi_{i o}^{t}$ to LHS and $\sum_{o \in C h\left(N^{t+1}\right)} \pi_{i o}^{t+1}$ to RHS, we obtain $\sum_{o \in \bar{X} \cup C h\left(N^{1, \cdots, t+1}\right)} \pi_{i o}^{t}<\sum_{o \in \bar{X} \cup C h\left(N^{1, \cdots, t+1}\right)} \pi_{i o}^{t+1}$. Altogether, for each $i \in N^{1, \cdots, t}$ and each $k \in N^{t+1}$, we have

$$
\begin{aligned}
\sum_{o \in \bar{X} \cup C h\left(N^{1, \cdots, t+1}\right)} \pi_{i o}^{t} & <\sum_{o \in \bar{X} \cup C h\left(N^{1, \cdots, t+1}\right)} \pi_{i o}^{t+1}=\sum_{o \in \bar{X} \cup C h\left(N^{1, \cdots, t+1}\right)} \pi_{k o}^{t+1} \\
& =\sum_{o \in \bar{X} \cup C h\left(N^{t+1}\right)} \pi_{k o}^{t+1} \leq \sum_{o \in \bar{X} \cup C h\left(N^{t+1}\right)} \pi_{k o}^{t},
\end{aligned}
$$

where the first equality comes from $s d$ no-envy, the second equality from Corollary 1(iii), and the last inequality from Lemma 6 . Thus, $\sum_{o \in \bar{X} \cup C h\left(N^{1, \cdots, t+1}\right)} \pi_{i o}^{t}<\sum_{o \in \bar{X} \cup C h\left(N^{t+1}\right)} \pi_{k o}^{t}$, in violation of $s d$ no-envy.

From the induction, we conclude that there is $\pi^{m} \in \Phi\left(R^{m}\right)$ such that for each $i \in N\left(=N^{1, \cdots, m}\right)$, $\sum_{o \in \bar{X} \cup C h\left(N^{1, \cdots, m}\right)} \pi_{i o}^{m}<\tau(m)<1$. Note that at $R^{m}$, each agent is indifferent among all objects in $N \backslash\left[\bar{X} \cup C h\left(N^{1, \cdots, m}\right)\right]$. By feasibility, each agent is assigned $(1-\tau(m))$ probability of receiving these objects at $E S\left(R^{m}\right)$, and $\left(1-\sum_{o \in \bar{X} \cup C h\left(N^{1, \cdots, m}\right)} \pi_{i o}^{m}\right)$ of receiving the same objects at $\pi^{m}$, respectively. Thus, $\pi^{m} \notin E S\left(R^{m}\right)$, and by Lemma $2, \pi^{m}$ is not sd-efficient at $R^{m}$.

\subsection{Independence of the Axioms in Theorem 1}

We establish the independence of the requirements listed in Theorem 1. In each of the following cases, we indicate which requirement is violated, and provide a correspondence which is not a subcorrespondence of the ES.

- $\boldsymbol{S d}$ efficiency: Consider the rule that assigns for each economy, each agent, and each object, the probability $\frac{1}{|A|}$ to the agent receiving the object.

- $\boldsymbol{S d}$ no-envy: For a strict ordering over the agents, consider the rule that maximizes agents' welfare lexicographically in that order. ${ }^{22}$

- Limited invariance: Let $N=\{1,2, \cdots,|N|\}, A \equiv\{a, \cdots, z\}$, and $R \in \mathcal{R}^{N}$ be such that (i) $R_{1}: a b c \cdots, R_{2}: a c b \cdots, R_{3}: b c a \cdots$, and (ii) the other agents rank $\{a, b, c\}$ lower than each object in $A \backslash\{a, b, c\}$ and have different most preferred objects. Let $\pi$ be such that $\pi_{1}=\left(\frac{1}{2}, \frac{1}{6}, \frac{1}{3}, 0, \cdots, 0\right), \pi_{2}=\left(\frac{1}{2}, 0, \frac{1}{2}, 0, \cdots, 0\right), \pi_{3}=\left(0, \frac{5}{6}, \frac{1}{6}, 0, \cdots, 0\right)$, and each other agent is assigned his most preferred object with probability 1 . Let $\Phi$ be the correspondence defined by setting $\Phi(R) \equiv\{\pi\}$ and for each $R^{\prime} \neq R, \Phi\left(R^{\prime}\right) \equiv E S\left(R^{\prime}\right)$.

Our result can be considered as an extension of the characterization in Hashimoto et al. (2013) to the weak preference domain: recall that on the strict preference domain, the extended serial correspondence coincides with the serial rule. ${ }^{23}$

\footnotetext{
${ }^{22}$ Roughly speaking, this is the serial dictatorship adapted to the weak preference domain.

${ }^{23}$ We use indifferences when we construct $R^{1}, \cdots, R^{m}$, but to modify the proof for the strict preferences domain,
} 


\section{Another Characterization}

We offer here another characterization of the ES correspondence, along the lines of Kesten et al. (2011). The requirements are a weaker efficiency requirement than weak sd-efficiency and a fairness requirement that we adapt from these authors. These requirements apply to each assignment matrix separately. Let $\pi \in \Pi$.

Non-wastefulness: for each $R \in \mathcal{R}^{N}$, each $i \in N$, and each pair $a, b \in A$,

$$
\text { if } \pi_{i a}>0 \text { and } b P_{i} a \text {, then } \sum_{j \in N} \pi_{j b}=1 \text {. }
$$

Ordinal Fairness: for each $R \in \mathcal{R}^{N}$, each pair $i, j \in N$, and each $a \in A$,

$$
\text { if } \pi_{i a}>0 \text {, then } \sum_{o \in U\left(R_{j}, a\right)} \pi_{j o}>\sum_{o \in U\left(R_{i}, a\right)} \pi_{i o} .
$$

Theorem 2. Let $R \in \mathcal{R}^{N}$. An assignment matrix is selected by ES for $R$ if and only if it is non-wasteful and ordinally fair at $R$.

Proof. We first present an alternative representation of assignments, which significantly simplifies the proof of the theorem. Let $P \in \mathcal{P}^{N}$ be a strict preference profile. Let $\pi \in \Pi$. For each $i \in N$, we represent $\pi_{i}$ as the endpoint of a process in which agents consume objects at unit speed in the decreasing order of $P_{i}$ over the time interval $[0,1]$; first, agent $i$ consumes his most preferred object at $P_{i}$, say $a$, from time 0 to time $\pi_{i a}$; second, he consumes his second most preferred object at $P_{i}$, say $b$, from time $\pi_{i a}$ to time $\left(\pi_{i a}+\pi_{i b}\right)$, and so on; he stops consuming objects at time 1 . Since the process is made in the decreasing order of his preferences, once he switches from one object to another, he never returns to the former object. ${ }^{24}$ For each $i \in N$, define the consumption schedule representing $\boldsymbol{\pi}_{\boldsymbol{i}}$ at $\boldsymbol{P}_{\boldsymbol{i}}$ as the process described above and denote it by $t\left(\pi_{i}, P_{i}\right)$. Let $t(\pi, P) \equiv\left(t\left(\pi_{i}, P_{i}\right)\right)_{i \in N}$ be the profile of consumption schedules representing $\boldsymbol{\pi}$ at $\boldsymbol{P}$. Let $a \in A, B \subseteq A$ and $\tau \in] 0,1]$. Given a profile of consumption schedules, we say that agent $\boldsymbol{i}$ stops consuming $\boldsymbol{B}$ at $\boldsymbol{\tau}$ if he switches from the least preferred object in $B$ to another object at time $\tau$ under his consumption schedule. We say that $\boldsymbol{B}$ reaches exhaustion at time $\tau$ if $\tau$ is the earliest time at which the total probabilities that the agents have consumed for the objects in $B$ equals $|B|$.

we can change this construction so that $R^{1}, \cdots, R^{m}$ are strict preferences. First, introduce a tie-breaking rule $\sigma_{0}$, common for all agents, and we use $P^{\sigma_{0}}\left(R^{1}\right), \cdots, P^{\sigma_{0}}\left(R^{m}\right)$, instead of $R^{1}, \cdots, R^{m}$. Second note that, throughout the proof of Theorem 1, we always make the statements on the "sum" of probabilities assigned to subsets of objects, like $\bar{X}, C h(i), C h\left(N^{1}\right)$, and $C h\left(N^{1, \cdots, t}\right)$. Thus, such a tie-breaking rule does not affect any argument, stated in terms of sum of probabilities of objects that are adjacent in preferences.

There are two exceptions, but they are modified as follows: (1) Case 1 in the proof of Lemma 2: we need to modify $U\left(\bar{R}_{i}^{t+1}, a\right)=\bar{X} \cup C h\left(N^{1, \cdots, k}\right)$ into $U\left(\bar{R}_{i}^{t+1}, a\right) \subseteq \bar{X} \cup C h\left(N^{1, \cdots, k}\right)$ and

$\sum_{o \in U\left(\bar{R}_{i}^{t+1}, a\right)} \pi_{i o}=\sum_{o \in \bar{X} \cup C h\left(N^{1, \cdots, k}\right)} \pi_{i o}$ into $\sum_{o \in U\left(\bar{R}_{i}^{t+1}, a\right)} \pi_{i o} \leq \sum_{o \in \bar{X} \cup C h\left(N^{1, \cdots, k}\right)} \pi_{i o} ;$ (2) the very last step in the proof of Theorem 1: instead of using feasibility alone, we need to invoke feasibility and sd no-envy to evaluate $E S\left(P^{\sigma_{0}}\left(R^{m}\right)\right)$ and $\pi^{m}$. At $R^{m}$, all agents have the same rankings over the objects other $\operatorname{than} \bar{X} \cup C h\left(N^{1, \cdots, m}\right)$. Therefore, by feasibility and $s d$ no-envy, they have to receive the same probability on each of these objects at $E S\left(P^{\sigma_{0}}\left(R^{m}\right)\right)$ and $\pi^{m}$, respectively. As before, we have $\pi^{m} \notin E S\left(P^{\sigma_{0}}\left(R^{m}\right)\right)$ and by Lemma 2 , we obtain the violation of sd-efficiency at $\pi^{m}$.

${ }^{24}$ For the formal definition, see Heo (2011). This representation is also used in Bogomolnaia and Heo (2012) and Heo (2013). 
Now, consider a weak preference profile, $R \in \mathcal{R}^{N}$. We introduce a profile of "tie-breakers" for indifferent objects, one for each agent; we then work with the strict preference profile induced from each weak preference profile. For each $i \in N$, let $\sigma_{i}$ be a bijection from $A$ to $\{1, \cdots,|A|\}$. Let $\Sigma$ be the set of all bijections. When agent $i$ finds two objects $a$ and $b$ indifferent, he breaks the tie by ranking $a$ above $b$ whenever $\sigma_{i}(a)<\sigma_{i}(b)$. For each $i \in N$, let $P^{\sigma_{i}}\left(R_{i}\right) \in \mathcal{P}$ be the strict preference induced from $R_{i}$ by using $\sigma_{i}$ : for each pair $a, b \in A, a P^{\sigma_{i}}\left(R_{i}\right) b$ if and only if either (i) $a P_{i} b$ or (ii) $a I_{i} b$ and $\sigma_{i}(a)<\sigma_{i}(b)$. Let $P^{\sigma}(R) \equiv\left(P^{\sigma_{i}}\left(R_{i}\right)\right)_{i \in N}$.

Let $R \in \mathcal{R}^{N}$. The following lemma states an important structural property of assignment matrices selected by the extended serial correspondence.

Lemma 7. Let $\pi \in \Pi$. For each $R \in \mathcal{R}^{N}$, the following statements are equivalent.

(i) $\pi \in E S(R)$.

(ii) for each $\sigma \in \Sigma^{N}$ and each $a \in A$, an agent stops consuming $U\left(R_{i}, a\right)$ at a time $\tau \in[0,1[$ only if $U\left(R_{i}, a\right)$ reaches exhaustion at $\tau$ under $t\left(\pi, P^{\sigma}(R)\right)$.

Proof. (i) $\rightarrow($ ii): This implication comes directly from the definition of ES.

(ii) $\rightarrow$ (i): Let $R \in \mathcal{R}^{N}$ and $\pi \in \Pi$ be the assignment matrix satisfying (ii). Let $\bar{m}$ be the last step of the ES algorithm for $R$. Suppose, by contradiction, that $\pi \notin E S(R)$. Then, there are $s \in\{1, \cdots, \bar{m}\}$ and $i \in N_{s}$ such that $\sum_{o \in U\left(R_{i}, C h\left(R_{i}, A_{s}\right)\right)} \pi_{i o}<\sum_{o \in U\left(R_{i}, C h\left(R_{i}, A_{s}\right)\right)} E S_{i o}(R)$. For each such agent $i$, let

$s(i) \equiv \underset{s \in\{1, \cdots, \bar{m}\}}{\operatorname{argmin}}\left\{\sum_{o \in U\left(R_{i}, C h\left(R_{i}, A_{s}\right)\right)} \pi_{i o}: \sum_{o \in U\left(R_{i}, C h\left(R_{i}, A_{s}\right)\right)} \pi_{i o}<\sum_{o \in U\left(R_{i}, C h\left(R_{i}, A_{s}\right)\right)} E S_{i o}(R)\right\},{ }^{25}$ and for each other agent $i$, let $s(i) \equiv \infty .^{26}$ Let $i^{*} \in \operatorname{argmin}_{i \in N} \sum_{o \in U\left(R_{i}, C h\left(R_{i}, A_{s(i)}\right)\right)} \pi_{i o}$ and $s^{*} \equiv s\left(i^{*}\right)$. If there are several such $i^{*}$, choose one with the largest $s^{*}$ (denote this condition by (1)). Let $\tau^{*} \equiv \sum_{o \in U\left(R_{i^{*}}, C h\left(R_{i^{*}}, A_{s^{*}}\right)\right)} \pi_{i^{*} o}$. Note that $\tau^{*}<\sum_{t=1}^{s^{*}} \lambda_{t}^{*}$ and for each $i \in N_{s^{*}}$, $\sum_{o \in U\left(R_{i}, C h\left(R_{i}, A_{s^{*}}\right)\right)} \pi_{i o} \geq \tau^{*}$. Let $\widehat{N} \equiv\left\{n \in N_{s^{*}}: \sum_{o \in U\left(R_{n}, C h\left(R_{n}, A_{s^{*}}\right)\right)} \pi_{n o}=\tau^{*}\right\}$ and $\widehat{X} \equiv$ $\bigcup_{n \in \widehat{N}} C h\left(R_{n}, A_{s^{*}}\right)$. Then, for each $n \in N_{s^{*}} \backslash \widehat{N}, \sum_{o \in U\left(R_{n}, C h\left(R_{n}, A_{s^{*}}\right)\right)} \pi_{n o}>\tau^{*}$ (denote this statement by $(2))$.

We claim that $\sum_{i \in \widehat{N}} \sum_{o \in \widehat{X}} \pi_{i o}=|\widehat{X}|$. Suppose otherwise; there are $j \in N \backslash \widehat{N}$ and $d \in \widehat{X}$ such that $\pi_{j d}>0$. We first show that $\sum_{o \in U\left(R_{j}, d\right)} \pi_{j o} \leq \tau^{*}$ (denote this statement by (3)). Otherwise, let $\sigma \in \Sigma^{N}$ be such that for each $n \in N, \sigma_{n}(d)=|A|$; there is $i \in \widehat{N}$ such that $d \in C h\left(R_{i}, A_{s^{*}}\right)$; note that $\sum_{o \in U\left(R_{i}, d\right)} \pi_{i o}=\tau^{*}<\sum_{o \in U\left(R_{j}, d\right)} \pi_{j o}$; agent $i$ stops consuming $d$ before this object reaches exhaustion, violating (ii) under $t\left(\pi, P^{\sigma}(R)\right)$. Now there are two cases.

Case 1. $j \in N_{s^{*}}$ : If $d \in C h\left(R_{j}, A_{s^{*}}\right)$, it follows from (2) that

$$
\sum_{o \in U\left(R_{j}, d\right)} \pi_{j o}=\sum_{o \in U\left(R_{j}, C h\left(R_{j}, A_{s^{*}}\right)\right)} \pi_{j o}>\tau^{*},
$$

\footnotetext{
${ }^{25}$ That is, $C h\left(R_{i}, A_{s(i)}\right)$ is the most preferred objects on which $\pi_{i}$ and $E S_{i}(R)$ "diverge" in welfare terms. Thus, $\pi_{i}$ coincides with an ES assignment for agent $i$ on $U\left(R_{i}, C h\left(R_{i}, A_{s(i)}\right) \backslash C h\left(R_{i}, A_{s(i)}\right)\right.$.

${ }^{26}$ That is, $s(i)$ is the earliest step of the ES algorithm, at which the assignment for agent $i$ "diverges" from the ES assignment.
} 
a contradiction to (3). Thus, $d \notin C h\left(R_{j}, A_{s^{*}}\right)$. Since $\pi_{j d}>0, \sum_{o \in U\left(R_{j}, C h\left(R_{j}, A_{s^{*}}\right)\right)} \pi_{j o}<$ $\sum_{o \in U\left(R_{j}, d\right)} \pi_{j o} \leq \tau^{*}$, and thus, $\left(i^{*}, s^{*}\right)$ should have not been chosen, a contradiction.

Case 2. $j \notin \boldsymbol{N}_{\boldsymbol{s}^{*}}$ : Let $\gamma$ be the smallest positive integer such that $j \in N_{s^{*}+\gamma}$. By definition of the ES solution, if an agent does not belong to a bottleneck set at a step, then at least one of his best available objects does not reach exhaustion at that step and remains available in the next step. Thus, since agent $j$ does not belong to a bottleneck set throughout steps $s^{*}, \ldots,\left(s^{*}+\gamma-1\right)$, each object in $C h\left(R_{j}, A_{s^{*}+\gamma}\right)$ is at least as desirable as each object in $A_{s^{*}}$. Thus, $C h\left(R_{j}, A_{s^{*}+\gamma}\right) R_{j} d$, which implies $\sum_{o \in U\left(R_{j}, C h\left(R_{j}, A_{s^{*}+\gamma}\right)\right)} \pi_{j o} \leq \sum_{o \in U\left(R_{j}, d\right)} \pi_{j o}$. From $(3), \sum_{o \in U\left(R_{j}, d\right)} \pi_{j o} \leq \tau^{*}\left(<\sum_{l=1}^{s^{*}} \lambda_{l}^{*}\right)$. From the definition of ES, $\sum_{l=1}^{s^{*}} \lambda_{l}^{*}<\sum_{l=1}^{s^{*}+\gamma} \lambda_{l}^{*}=$ $\sum_{o \in U\left(R_{j}, C h\left(R_{j}, A_{s^{*}+\gamma}\right)\right)} E S_{j o}(R)$. Altogether,

$$
\sum_{o \in U\left(R_{j}, C h\left(R_{j}, A_{s^{*}+\gamma}\right)\right)} \pi_{j o} \leq \tau^{*}<\sum_{o \in U\left(R_{j}, C h\left(R_{j}, A_{s^{*}+\gamma}\right)\right)} E S_{j o}(R) .
$$

If $C h\left(R_{j}, A_{s^{*}+\gamma}\right) P_{j} d$, then $\sum_{o \in U\left(R_{j}, C h\left(R_{j}, A_{s^{*}+\gamma}\right)\right)} \pi_{j o}<\sum_{o \in U\left(R_{j}, d\right)} \pi_{j o} \leq \tau^{*}$. Thus, $\left(i^{*}, s^{*}\right)$ should have not been chosen, a contradiction. If $C h\left(R_{j}, A_{s^{*}+\gamma}\right) I_{j} d$, then $\sum_{o \in U\left(R_{j}, C h\left(R_{j}, A_{s^{*}+\gamma}\right)\right)} \pi_{j o}$ $=\sum_{o \in U\left(R_{j}, d\right)} \pi_{j o}\left(\leq \tau^{*}\right)$. There are two subcases. First, $s(j)=s^{*}+\gamma$. By $(1),\left(i^{*}, s^{*}\right)$ should have not been chosen, a contradiction. Second, $s(j)<s^{*}+\gamma$. Since $j \in N_{s(j)} \cap$ $N_{s^{*}+\gamma}$, we have $C h\left(R_{j}, A_{s(j)}\right) P_{j} C h\left(R_{j}, A_{s^{*}+\gamma}\right)$. Since $\pi_{j d}>0, \sum_{o \in U\left(R_{j}, C h\left(R_{j}, A_{s(j)}\right)\right)} \pi_{j o}<$ $\sum_{o \in U\left(R_{j}, C h\left(R_{j}, A_{s^{*}+\gamma}\right)\right)} \pi_{j o} \leq \tau^{*}$. Thus, $\left(i^{*}, s^{*}\right)$ should have not been chosen, a contradiction.

Altogether, $\widehat{N} \subseteq N_{s^{*}}$ and $\pi \in \Pi$ is such that

- there is $\bar{\pi} \in E S(R)$ such that $\pi$ coincides with $\bar{\pi}$ on $\bigcup_{i \in \widehat{N}} U\left(R_{i}, C h\left(R_{i}, A_{s^{*}}\right)\right) \backslash C h\left(R_{i}, A_{s^{*}}\right)$,

- for each $i \in \widehat{N}, \sum_{o \in U\left(R_{i}, C h\left(R_{i}, A_{s^{*}}\right)\right)} \pi_{i o}=\tau^{*}<\sum_{l=1}^{s^{*}} \lambda_{l}^{*}$,

- $\sum_{i \in \widehat{N}} \sum_{o \in C h\left(R_{i}, A_{s^{*}}\right)} \pi_{i o}=\sum_{i \in \widehat{N}} \sum_{o \in \widehat{X}} \pi_{i o}=|\widehat{X}|$.

There are two possibilities. First, $\widehat{N} \subsetneq N_{s^{*}}$. Then, $\widehat{X} \subsetneq X_{s^{*}}$ and $\widehat{N}$ is the largest bottleneck set at some step $s<s^{*}$ at which $\bar{X}$ reaches exhaustion, a contradiction. Second, $\widehat{N}=N_{s^{*}}$. Since $N_{s^{*}}$ is the largest bottleneck set at step $s^{*}, \sum_{l=1}^{s^{*}} \lambda_{l}^{*}=\tau^{*}$, a contradiction.

Now, we complete the proof of the theorem. Let $R \in \mathcal{R}^{N}$. Suppose that $\pi \in E S(R)$. From Lemma 7 , it follows that $\pi$ satisfies the two requirements. Conversely, let $\pi$ be an assignment matrix satisfying the two requirements. Suppose, by contradiction, that $\pi \notin E S(R)$. Then, there is $\sigma \in \Sigma^{N}$ such that $t\left(\pi, P^{\sigma}(R)\right)$ is as follows: there are $i \in N, a \in N$, and $\tau \in[0,1[$ such that agent $i$ stops consuming some $b \in U\left(R_{i}, a\right)$ at some time $\tau$ at which $U\left(R_{i}, a\right)$ has not reached exhaustion. By non-wastefulness, there is $j \in N$ who consumes a positive probability of $b$ after $\tau$ at $t\left(\pi, P^{\sigma}(R)\right)$. However, $\sum_{o \in U\left(R_{i}, b\right)} \pi_{i o}<\sum_{o \in U\left(R_{j}, b\right)} \pi_{j o}$, in violation of ordinal fairness.

\section{References}

[1] Birkhoff, G., 1946. Three observations on linear algebra. Revi. Univ. Nac. Tucuma. ser A. $5,147-151$. 
[2] Bogomolnaia, A., Heo E.-J., 2012. Probabilistic assignment of objects: characterizing the serial rule. J. Econ. Theory. 147, 2072-2082.

[3] Bogomolnaia, A., Moulin, H., 2001. A new solution to the random assignment problem. J. Econ. Theory. 100, 295-328.

[4] Gibbard. A., 1978. Straightforwardness of game forms with lotteries as outcomes. Econometrica. 46, 595-614.

[5] Hashimoto, T., Hirata, D., 2011. Characterizations of the probabilistic serial mechanism. mimeo

[6] Hashimoto, T., Hirata, D., Kesten, O., Kurino, M., Ünver, U., 2013. Two axiomatic approaches to the probabilistic serial mechanism. Theor. Econ. forthcoming.

[7] Heo, E.-J., 2011. Random assignment problem with multiple demands: a generalization and a characterization of the serial rule. mimeo

[8] Heo, E.-J., 2013. The extended serial correspondence on the rich preference domain. Int. J. Game. Theor. forthcoming.

[9] Heo, E.-J. Yılmaz, Ö., 2011. A characterization of the extended serial correspondence. mimeo

[10] Katta, A.-K., and Sethuraman, J., 2006. A solution to the random assignment problem on the full preference domain. J. Econ. Theor. 131, 231-250.

[11] Kesten, O., Kurino, M., Ünver, U., 2011. Fair and efficient assignment via the probabilistic serial mechanism. mimeo

[12] Thomson, W., 2010a. Consistency for the probabilistic assignment of objects. mimeo

[13] Thomson, W., 2010b. Strategy-proof allocation rules. mimeo

[14] Von Neumann, J. 1953. A certain zero-sum two-person game equivalent to the optimal assignment problem. In: Kuhn HW, Tucker AW (ed) Contributions to the theory of games, vol. II, Princeton University Press, Princeton, pp 5-12. 